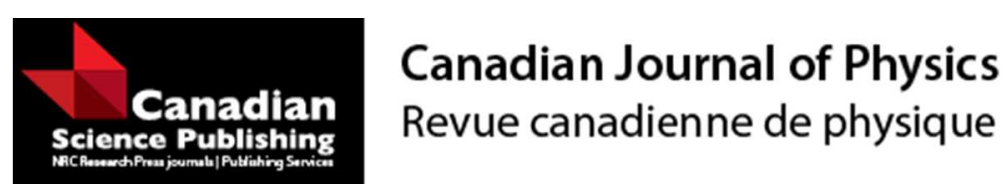

\title{
Comparison of errors between a differential and a classical abundance analysis
}

\begin{tabular}{|r|l|}
\hline Journal: & Canadian Journal of Physics \\
\hline Manuscript ID & cjp-2016-0877.R1 \\
\hline Manuscript Type: & Article \\
\hline Date Submitted by the Author: & 13 -Feb-2017 \\
\hline Keyword: & $\begin{array}{l}\text { Precision Spectroscopy, Differential chemical analysis, Stellar chemical } \\
\text { abundances, Stellar parameters, Chemical evolution }\end{array}$ \\
\hline Please Select from this Special & $\begin{array}{l}\text { Reggiani, Henrique; Universidade de Sao Paulo, Instituto de Astronomia, } \\
\text { Melendez, Jorge; Universidade de Sao Paulo, Astronomia }\end{array}$ \\
\hline Issues list if applicable: & ASOS \\
\hline & \multicolumn{2}{|c|}{} \\
\hline
\end{tabular}




\title{
Comparison of errors between a differential and a classical abundance analysis
}

\author{
Henrique Reggiani and Jorge Meléndez \\ Universidade de São Paulo - Instituto de Astronomia, Geofísica e Ciências \\ Atmosféricas \\ hreggiani@gmail.com
}

\begin{abstract}
The differential abundance analysis method can improve the precision of stellar chemical abundances. The method compares the equivalent widths of a certain line in a star with the same line in a star considered to be a standard representative of its class, using high resolution and high $\mathrm{S} / \mathrm{N}$ spectra. The method has achieved great results by reducing the measurements errors to unprecedent low levels. However, to date, there has not been a consistent analysis on the actual improvements of this method when compared to a classical analysis in metal-poor stars. Here we present a comparison between the errors of a classical stellar analysis and a differential analysis among low metallicity stars.
\end{abstract}

Keywords: Precision spectroscopy; Differential chemical analysis; Stellar abundances; Stellar parameters; Chemical evolution

\section{Introduction}

In the last few years different authors have used the differential abundance analysis to evaluate stellar evolution effects $[6,18]$, to recognize planet signatures in chemical abundances of stars $[3,10,17,1]$, to study the chemical evolution of the solar neighborhood $[9,14]$, study abundance anomalies in stellar clusters [19] and distinct populations in the stellar halo $[8,11,2]$. Using high resolution and high S/N spectra previous works have been able to achieve errors as low as 0.004 dex for solar twins [17] and 0.02 dex for metal-poor stars [14]. But it is, so far, unclear whether these low errors are entirely due to the analysis technique, and how much the spectral quality influences the precision achieved. Here we have consistently analyzed the errors of a classical and a differential analysis in two samples of metal-poor stars with different spectral quality.

\section{Samples and method of analysis}

We have analyzed two separate samples. The first sample is composed by the two stars previously described in [14] (Keck spectra with $\mathrm{R} \approx 95000$ and $\mathrm{S} / \mathrm{N}=400$ at $500 \mathrm{~nm}$ ). The second sample is composed by 11 stars observed with UVES/VLT 
$(\mathrm{R} \approx 50000$ and $\mathrm{S} / \mathrm{N}=180$ at $500 \mathrm{~nm})$. The UVES spectra were reduced through the ESO pipeline and further corrections (such as barycentric and radial velocity correction) were done using the IRAF package for python, pyraf, and the spectra were normalized via IRAF (using the same polynomial and polynomial orders when possible). In both samples we measured equivalent widths using the splot task in IRAF.

The line lists used for the analysis were constructed using the VALD ([15]) database along with new $\log (\mathrm{gf})$ values and hyperfine structure data from $\mathrm{Ku}$ rucz $^{1}$. We calculated the stellar parameters and chemical abundances in two manners. First we have performed a classical spectroscopic analysis of the data. We defined the stellar parameters through excitation equilibrium of the $\mathrm{Fe} I$ and Fe II lines to determine $\mathrm{T}_{\text {eff }}$, obtained $\log \mathrm{g}$ through ionization equilibrium, and microturbulent velocity by allowing no trend in line abundances with reduced EW. This was performed using the q2 semi automated program [12], which uses the radiative transfer code MOOG [16] to calculate the best solution for the parameters and the errors for each parameter via statistical analysis. After that we calculate all the chemical abundances using q2 and, once again, the program calculates the errors using observational errors and the stellar parameters errors.

We also calculated these quantities using a line-by-line differential analysis, as described in $[5,19,13]$. The differential method compares the chemical abundance of each line in a given star, with the same line of a standard star. This way, error sources cancel out, in particular the $g f$ values. The absolute abundances can be written as: $\log \frac{W}{\lambda}=B+A_{x}+\log (g f)+\log (\lambda)-\theta_{X e x c}-\log k_{\text {cont }}$, and the differential abundances are: $\log \frac{W_{1, i}}{W_{2, i}}=A_{x, i}^{1}-A_{x, i}^{2}-\left(\theta^{1}-\theta^{2}\right)_{X e x c}-\log \frac{k_{c o n t}^{1}}{k_{\text {cont }}^{2}}$. We then compare these differential abundances of Fe I to calculate a differential excitation equilibrium and obtain a differential estimation of the $T_{\text {eff }}$, a differential ionization equilibrium between Fe I and Fe II lines to obtain $\log g$, and microturbulence by allowing no trend between differential line abundances with reduced EW. Note that in order to calculate the differential parameters of any given star it is necessary to know the stellar parameters of the standard star beforehand with the best possible accuracy and precision.

In our work the standard star was selected due to the availability of stellar parameters from non-spectroscopic sources with low errors, $\mathrm{T}_{\text {eff }}$ from the IRFM [4], and $\log g$ from [7], who estimate the surface gravity using the absolute magnitude of the star from fundamental relation $\log _{\frac{g}{\log g_{\odot}}}=\log \frac{M}{M_{\odot}}+$ $4 \log \frac{\mathrm{T}_{\text {eff }}}{\mathrm{T}_{\text {eff }, \odot+0.4\left(\mathrm{M}_{\mathrm{bol}}-\mathrm{M}_{\mathrm{bol}, \odot)}\right)}}$, which have errors of $50 \mathrm{~K}$ and 0.15 dex respectively. Using the Fe I and Fe II lines of these stars as reference we performed a line by line differential analysis on the other stars and estimated their stellar parameters, abundances and their respective errors. The effective temperature, $\log g$, microturbulent velocity and metallicity of the remaining stars were calculated with the differential method, as explained above

Stellar parameters calculated strictly from spectroscopic methods are known to differ from photometric methods in metal-poor stars; usually spectroscopic

\footnotetext{
$\overline{{ }^{1} \text { http://kurucz.harvard.edu/linelists.html }}$
} 
temperatures are $\approx 300 \mathrm{~K}$ lower than photometric ones. The effective temperature of star G64-12, for example, is $6168 \mathrm{~K}$ when estimated through excitation equilibrium and $6463 \mathrm{~K}$ from the IRFM[4], and the temperature of star G64-37 is $6258 \mathrm{~K}$ from classical excitation equilibrium. Interestingly, the temperature of this star is $6568 \mathrm{~K}$ from a differential equilibrium, using star G64-12 with IRFM temperature as the standard point of comparison. The surface gravity experiences more extreme differences, with relative differences up to 1 dex, between the classical ionization equilibrium to the $\log g$ from the absolute magnitude, and, thus it shows the same large departure when compared to the differential ionization with a standard star with $\log g$ from the absolute magnitude. The use of these different sets of stellar parameters (strictly spectroscopic vs differential analysis with a standard star with IRFM temperature and log $g$ from absolute magnitude) will also translate into different abundances results, usually a difference on the order of 0.15 dex. As the stellar parameters and abundances results differ when using a classical and differential analysis, we will not focus on their values. We are only interested in the ratio between the errors of the classical analysis and the differential analysis.

All the abundance errors presented here include the observational errors (defined as $\frac{\sigma}{\sqrt{N}}$ ) added in quadrature to the quadrature of the errors due to the stellar parameters errors.

\section{Results and Discussion}

In Fig. 1a we show the $\mathrm{T}_{\text {eff }}$ error ratios for all the stars. In this figure the first star is from the Keck sample (G64-37). We do not compare errors of the standard stars of our samples because they come from different methods ( $\mathrm{T}_{\text {eff }}$ from the IFRM, and $\log g$ from the absolute magnitude).

There is a clear gain, specially for star G64-37 which is one of the Keck sample stars, which have the highest $\mathrm{S} / \mathrm{N}$ and resolution. The average gain on $\mathrm{T}_{\text {eff }}$ is 1.4 , the average gain for $\log \mathrm{g}$ was 1.3 and for microturbulence it was 1.6. In Figs. $1 b$ and 1c we show the gain on precision for the abundances (Fig $1 \mathrm{~b}$ shows the result for the Keck sample and Fig. 1c shows the average gain for each element throughout the entire UVES sample). As can be seen, the average gain for the Keck sample is twice the average gain for the UVES sample. We attribute this difference to the quality of the spectra. The better spectral quality will allow us to measure the EW more consistently between the two stars, which will make the comparison more precise, thus lowering the error bars. Overall it is clear that the differential analysis provides better errors when compared to a classical analysis.

We also compared the overall effect of the stellar parameters analysis in the final chemical abundance errors and concluded that there is no big benefit from a differential analysis if it is carried out with a classic spectroscopic calculation of the stellar parameters (average gain of 1.4 and 1.1 for the Keck and UVES samples respectively). 


\section{Conclusions}

With this simple, but consistent analysis, we were able to see that a differential analysis can increase the precision of our results by up to three times on average, depending on the quality of the spectra and measurements. We have also seen that the most important part of our analysis was the precise determination of the stellar parameters (the better constrained the stellar parameters the better our final abundance results).

\section{References}

1. K. Biazzo,R. Gratton, S. Desidera, et al., A\&A, A135, 583, 2015

2. C. K. Fishlock, D. Yong, et al., MNRAS, 57F, 2017

3. J. Meléndez, M. Asplund, B. Gustafsson, \& D. Yong, AJ, L66, 704, 2009

4. J. Meléndez, L. Casagrande, I. Ramírez, et al., A\&A, L3, 515, 2010

5. J. Meléndez, M. Bergemann, J.G. Cohen, et al., A\&A, A29, 543, 2012

6. T. Monroe, J. Meléndez, I. Ramírez, et al., AJ, L32, 774, 2013

7. P. E. Nissen, C. Akerman, M. Asplund, et al., A\&A, 319, 469, 2007

8. P.E. Nissen \& W. Schuster, A\&A, L10, 511, 2010

9. P. E. Nissen, A\&A, A52, 579, 2015

10. I. Ramírez, J. Meléndez \& M. Asplund, A\&A, L17, 508, 2009

11. I. Ramírez, J. Meléndez \& J. Chanamé, ApJ, 757, 164, 2012

12. I. Ramírez, J. Meléndez, J. Bean, et al., A\&A, A48, 572, 2014

13. I. Ramírez, S. Khanal \& P. Aleo, ApJ, 808, 13, 2015

14. H. Reggiani, J. Meléndez, et al., A\&A, A67, 586, 2016

15. T, Ryabchikova et al., 2015, PhyS, 90e4005R

16. C. A. Sneden, Ph.D. Thesis, Univ. Texas, 1973

17. M. Tucci Maia, J. Meléndez \& I. Ramírez, AJ, L25, 790, 2014

18. M. Tucci Maia, J. Meléndez, M. Castro, et al., A\&A, L10, 576, 2015

19. D. Yong, J. Meléndez, L. Grundah, et al., MNRAS, 434, 3542, 2013 


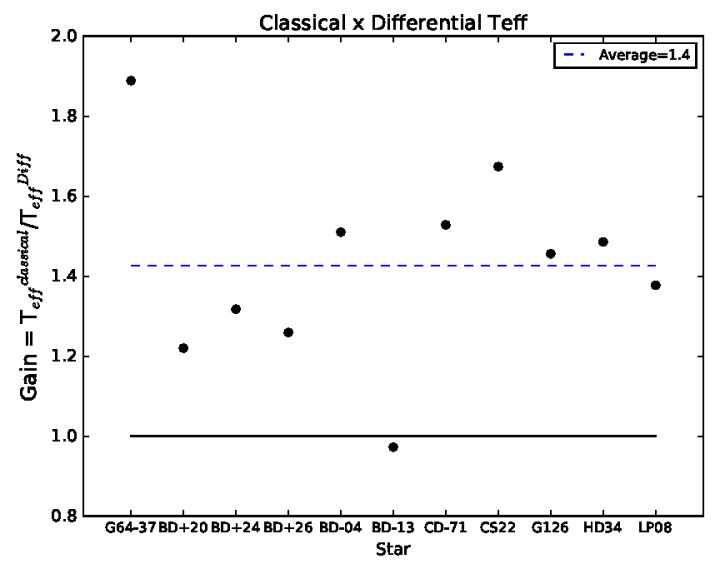

(a) Ratio between $T_{\text {eff }}$ errors, classical spectrocopic analysis / differential analysis, of all the stars in both samples. G64-37 is the star from the Keck sample.

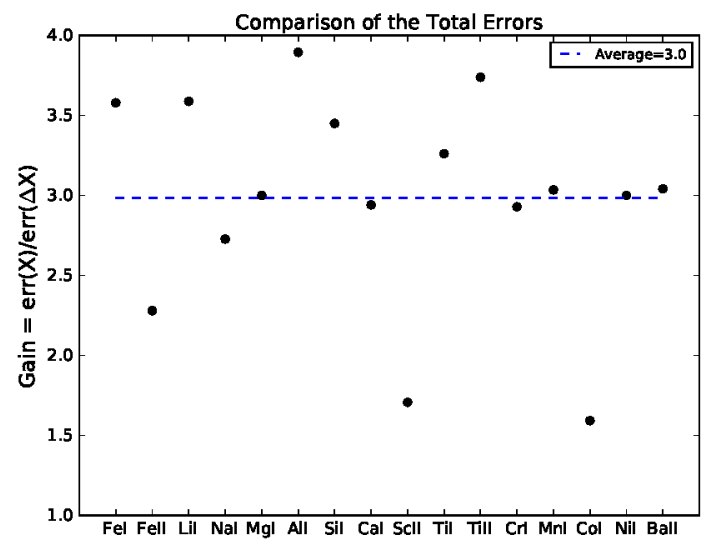

(b) Ratio between errors for chemical abundances (classical analysis/differential analysis) of the Keck sample.

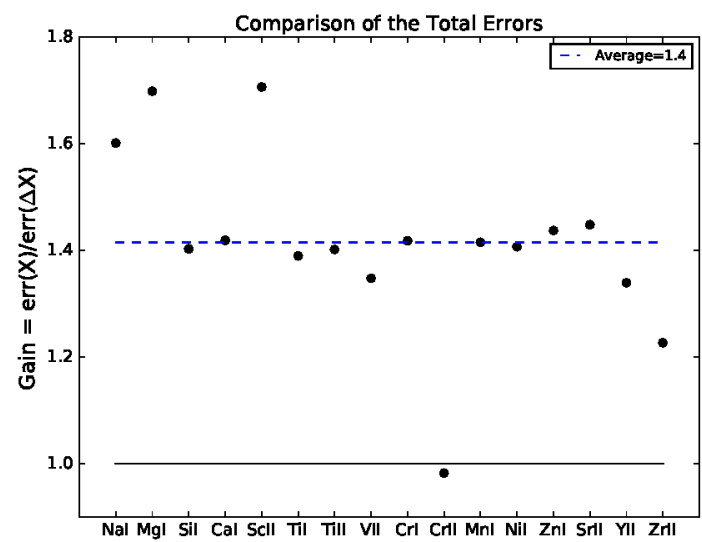

(c) Ratio between errors for chemical abundances (classical analysis/differential analysis) of the UVES sample. 